\title{
Effects of PYRIN-containing Apaf1-like protein 1 on isoflurane-induced postoperative cognitive dysfunction in aged rats
}

\author{
XIAONA ZHANG ${ }^{1}$, XIUSHUANG FAN $^{1}$, FAN LI $^{1}$, JINPENG QIU $^{1}$ and YANG ZHANG ${ }^{2}$ \\ Departments of ${ }^{1}$ Anesthesiology and ${ }^{2}$ Neurosurgery, The First Hospital of Jilin University, Changchun, Jilin 130021, P.R. China
}

Received October 10, 2019; Accepted February 5, 2020

DOI: $10.3892 / \mathrm{mmr} .2020 .11244$

\begin{abstract}
Postoperative cognitive dysfunction (POCD) is a prevalent neurocognitive disorder following surgery and anesthesia, particularly in elderly patients. Isoflurane is a widely used anesthetic agent, which is associated with the development of POCD; however, the precise mechanisms remain unclear. In the present study, aged rats were exposed to $2 \%$ isoflurane to establish a POCD model. The expression of PYRIN-containing Apaf1-like protein 1 (PYPAF1) was knocked down using a lentivirus containing specific short hairpin RNA. Subsequently, the spatial learning ability of rats was assessed using the Morris water maze. In addition, mRNA and protein expression levels were detected using reverse transcription-quantitative $\mathrm{PCR}$ and western blot analysis, respectively. Immunofluorescence double staining was also used to determine the expression of PYPAF1 and Iba-1 in the hippocampus. Neural apoptosis was observed using TUNEL-NeuN double staining. The results revealed that isoflurane exposure impaired the spatial learning ability of rats, while PYPAF1 knockdown alleviated cognitive impairment. In addition, isoflurane exposure induced activation of the PYPAF1 inflammasome, as evidenced by elevated expression of PYPAF1 and apoptosis-associated speck-like protein containing a caspase recruitment domain, while silencing of PYPAF1 partially reversed this effect. Furthermore, isoflurane exposure promoted the activation of microglia and caspase-1, and the secretion of interleukin (IL)-1 $\beta$ and IL-18, all of which were alleviated following PYPAF1 silencing. Moreover, isoflurane exposure induced neuronal apoptosis, elevated the levels of Bax and cleaved caspase-3, and inhibited the expression of Bcl-2; all of these effects were partially abrogated following PYPAF1 silencing. In conclusion, the results of the present study indicated that PYPAF1 silencing partially abolished
\end{abstract}

Correspondence to: Dr Yang Zhang, Department of Neurosurgery, The First Hospital of Jilin University, 71 Xinmin Avenue, Changchun, Jilin 130021, P.R. China

E-mail: zhangyang_8186@tom.com

Key words: postoperative cognitive dysfunction, isoflurane, PYPAF1, aged rats isoflurane-induced cognitive impairment, neuroinflammation and neuronal apoptosis. Therefore, PYPAF1 may be a potential therapeutic target for treatment of POCD.

\section{Introduction}

Postoperative cognitive dysfunction (POCD) is a type of postoperative complication that occurs in the central nervous system (CNS) $(1,2)$. POCD is regarded as a mild neurocognitive disorder without a formal definition $(3,4)$ and the diagnosis requires a validated neuropsychological test battery (5). POCD occurs in the first few weeks following surgery; it severely impairs cognitive function and increases mortality in patients who are affected (6). POCD is now considered to be a public health concern, which has a negative impact on the quality of life of patients who are affected and causes a high burden for social medical care (7). According to data from the Ministry of Civil Affairs of China, in 2018, the elderly population (>60 years old) reached 249.49 million, accounting for $17.9 \%$ of the total population of China (8). Thus, POCD may become a major health issue, due to lack of efficient diagnosis in the coming decades (9). Isoflurane-inhaled anesthesia was reported to be associated with the development of POCD by influencing brain activity, yet the exact mechanism has not been elucidated (10). Therefore, a deeper understanding of the role that isoflurane plays in the development of POCD is of great importance to discover novel approaches to eliminate isoflurane-induced cognitive impairment.

Increasing evidence has demonstrated that neuroinflammation is critical in the pathogenesis of neurodegenerative diseases, including Alzheimer's disease (AD), Parkinson's disease and POCD $(11,12)$. Surgery and anesthesia have been reported to induce neuroinflammatory responses and promote the secretion of proinflammatory cytokines, such as interleukin (IL)-1 $\beta$, IL- 6 and tumor necrosis factor- $\alpha$, which further activate microglia to an inflammatory phenotype $(13,14)$. Microglia, innate immune cells of the brain, serve a crucial role in brain homeostasis. Activation of microglia is considered a negative event and it is involved in the development of neurocognitive disorders, including POCD $(15,16)$. Furthermore, exposure to isoflurane has been shown to impair the spatial learning memory of aged mice, accompanied by activation of microglia and neuroinflammation (17). Yet the precise mechanisms remain unclear. 
Inflammasomes are cytosolic protein complexes assembled by pattern recognition receptors (PRRs), pathogen-associated molecular patterns and danger-associated molecular patterns (18). To date, five inflammasomes have been discovered, among which the PYRIN-containing Apaf1-like protein 1 (PYPAF1) inflammasome is the most investigated (19). The PYPAF1 inflammasome is comprised of a sensor protein PYPAF1, apoptosis-associated speck-like protein containing a caspase recruitment domain (ASC), aspartate-specific proteases and pro-caspase-1. PYPAF1 interacts with ASC, induces the activation of NF- $\mathrm{KB}$ and regulates inflammatory-associated processes (20). The PYPAF1 inflammasome activates the protease caspase-1, promotes the secretion of IL-1 $\beta$ and IL-18, and further induces neuroinflammation (21). Studies have shown that PYPAF1 may contribute to neuroinflammation in numerous cognitive impairment-related diseases (19), and inhibition of PYPAF1 activation has been found to inhibit inflammation in the brain (22); however, to the best of our knowledge, its role in POCD has not been elucidated.

In our preliminary studies, PYPAF1 was upregulated in the hippocampus of POCD rats. As PYPAF1 is associated with inflammation, it was hypothesized that PYPAF1 may have an important role in the development of POCD. The aim of the present study was to determine the possible mechanisms of POCD by examining PYPAF1 and inflammation, using a rat POCD model via isoflurane exposure. In addition, the role that PYPAF1 plays in microglial activation and neuroinflammation was investigated.

\section{Materials and methods}

Rat POCD model. A total of 138 healthy 20-month-old male Sprague Dawley (SD) rats (weight, 500-520 g; Liaoning Changsheng Biotechnology Co. Ltd.) were randomly divided into four groups: Control; isoflurane; isoflurane + short hairpin (sh)RNA-PYPAF1; and isoflurane + shRNA-negative control (NC). Rats were maintained in a standard experimental rodent room under a 12-h light/dark circle, at a temperature of $23 \pm 1^{\circ} \mathrm{C}$ and relative humidity of $50 \pm 1 \%$, with free access to water and food. The animals were adaptively fed for 1 week before establishment of the animal model. For the isoflurane + shRNA-PYPAF1 group, rats were injected with $5 \mu 1$ lentivirus containing PYPAF1-shRNA $\left[10^{8}\right.$ transduction units $\left.(\mathrm{TU}) / \mathrm{ml}\right]$ into the right lateral ventricle; for the isoflurane + shRNA-NC group, rats were injected with $5 \mu$ l lentivirus carrying NC-shRNA $\left(10^{8} \mathrm{TU} / \mathrm{ml}\right)$ into the right lateral ventricle. A total of 3 days after lentiviral injection, rats in the isoflurane, isoflurane + shRNA-PYPAF1 and isoflurane + shRNA-NC groups were maintained in a chamber prefilled with $2 \%$ isoflurane in $100 \%$ oxygen for $4 \mathrm{~h}$, rats in the control group were given $100 \%$ oxygen for $4 \mathrm{~h}$ (gas flow rate, $2 \mathrm{l} / \mathrm{min}$ ) (23). A total of 18 rats in each group were randomly selected and euthanized by intraperitoneal injection of $200 \mathrm{mg} / \mathrm{kg}$ sodium pentobarbital on day 3 , and six rats in each group were euthanized on day 7 following isoflurane exposure, and the hippocampi were collected. Hippocampi from 12 rats euthanized on day 3 and six rats euthanized on day 7 were cryopreserved in liquid nitrogen for molecular biological analysis. Hippocampi from six rats euthanized on day 7 were fixed with $4 \%$ paraformal- dehyde at $4{ }^{\circ} \mathrm{C}$ overnight for histological examinations. The other six rats in each group were used for behavioral testing. Another 18 SD rats were randomly divided into three groups (control, shRNA-PYPAF1 or shRNA-NC) for the assessment of transfection efficiency. All animal experiments were performed at the animal center at the First Hospital of Jilin University (Changchun, China) following the guidelines for the care and use of laboratory animals (24), and this study was approved by the First Hospital of Jilin University.

Construction of PYPAF1 shRNA lentiviral vectors. To generate PYPAF1-targeting shRNA expressing plasmids, double-stranded oligonucleotides encoding PYPAF1 shRNA were amplified in a system containing $5 \mathrm{X}$ oligo annealing buffer (1 mM EDTA, $50 \mathrm{mM} \mathrm{NaCl}, 10 \mathrm{mM}$ Tris), sense and antisense primers containing the PYPAF1 shRNA sequence (Sangon Biotech Co., Ltd.) using the following reaction conditions: $95^{\circ} \mathrm{C}$ for $5 \mathrm{~min}$, $90^{\circ} \mathrm{C}$ for $5 \mathrm{~min}, 85^{\circ} \mathrm{C}$ for $5 \mathrm{~min}$ and $80^{\circ} \mathrm{C}$ for $5 \mathrm{~min}$, followed by overnight annealing at room temperature. The primer sequences were as follows: Forward, 5'-ceggcccGGCTATGTACTATCT GCTAttcaagagaTAGCAGATAGTACATAGCCttttt-3' and reverse, 5'-aattaaaaaGGCTATGTACTATCTGCTAtctcttgaa TAGCAGATAGTACATAGCCggg-3'. After the Tet-pLKO-puro lentiviral vector (cat. no. 21915; Addgene, Inc.) was linearized with FastDigest AgeI and FastDigest EcoRI (cat. nos. FD1464 and FD0274; Thermo Fisher Scientific, Inc.), the oligonucleotides encoding PYPAF1 shRNA were inserted into the lentiviral vector with T4 DNA Ligase (cat. no. 2011A; Takara Biotechnology Co., Ltd.). The recombinant plasmids were then transformed into Escherichia coli DH5a-competent cells (cat. no. 9057; Takara Biotechnology Co., Ltd.). The plasmids were extracted from the positive clones using a Plasmid Maxi Preparation kit (cat. no. DP2802; BioTeke Corporation). A total of $24 \mathrm{~h}$ prior to transfection, $293 \mathrm{~T}$ cells were plated into a $10-\mathrm{cm}$ plate at density of $6 \times 10^{6}$. Tet-pLKO-puro-PYPAF1 shRNA lentiviral vectors ( $3 \mu \mathrm{g})$, packaging plasmid psPAX2 $(5 \mu \mathrm{g}$; cat. no. 12260; Addgene, Inc.) and envelop plasmid pMD2.G (5 $\mu \mathrm{g}$; cat. no. 12259; Addgene, Inc.) were co-transfected into 293T cells (Procell Life Science \& Technology Co., Ltd.) using Lipofectamine ${ }^{\circledR} 3000$ (cat. no. L3000-008; Invitrogen; Thermo Fisher Scientific, Inc.) at $37^{\circ} \mathrm{C}$ for $48 \mathrm{~h}$ to perform viral packaging. Lentivirus-containing supernatants were then centrifuged at $4,000 \mathrm{xg}$ at $4{ }^{\circ} \mathrm{C}$ for $10 \mathrm{~min}$, filtered through a $0.45-\mu \mathrm{m}$ filter and centrifuged at $4,000 \mathrm{x} \mathrm{g}$ at $4^{\circ} \mathrm{C}$ for $5 \mathrm{~min}$. The lentivirus stock was collected and stored at $-80^{\circ} \mathrm{C}$. The shRNA sequences were as follows: PYPAF1 shRNA, sense, 5'-CCGGCCCGGCT ATGTACTATCTGCTATTCAAGAGATAGCAGATAGTACA TAGCCTTTTT-3' and antisense, 5'-AATTAAAAAGGC TATGTACTATCTGCTATCTCTTGAATAGCAGATAGTAC ATAGCCGGG-3'; and NC-shRNA, sense, 5'-CCGGCCCTT CTCCGAACGTGTCACGTTTCAAGAGAACGTGACACGT TCGGAGAATTTTT-3' and antisense, 5'-AATTAAAAATTC TCCGAACGTGTCACGTTCTCTTGAAACGTGACACGTT CGGAGAAGGG-3'.

Reverse transcription-quantitative PCR (RT-qPCR). Hippocampal tissues were lysed and total RNA was extracted using commercial RNAprep pure kit (Tiangen Biotech Co., Ltd.). Total RNA was subsequently reverse transcribed into cDNA using oligo $(\mathrm{dT})_{15}$, dNTP, M-MLV and RNase inhibitor 
(Tiangen Biotech Co., Ltd.). The RT conditions were as follows: $25^{\circ} \mathrm{C}$ for $10 \mathrm{~min}, 42^{\circ} \mathrm{C}$ for $50 \mathrm{~min}$ and $80^{\circ} \mathrm{C}$ for $10 \mathrm{~min} . \mathrm{qPCR}$ was performed using $2 \mathrm{X}$ Power Taq PCR MasterMix (BioTeke Corporation) and SYBR-Green (Beijing Solarbio Science and Technology Co., Ltd.) on an Exicycler ${ }^{\mathrm{TM}} 96$ RT-PCR system (Bioneer Corporation). The thermocycling conditions were as follows: Initial denaturation at $94^{\circ} \mathrm{C}$ for $5 \mathrm{~min}$, followed by 40 cycles at $94^{\circ} \mathrm{C}$ for $10 \mathrm{sec}, 60^{\circ} \mathrm{C}$ for $20 \mathrm{sec}$ and $72^{\circ} \mathrm{C}$ for $30 \mathrm{sec}$, and a final extension step at $72^{\circ} \mathrm{C}$ for $6 \mathrm{~min}$. The data were analyzed using the $2^{-\Delta \Delta \mathrm{Cq}}$ method (25). GAPDH was used as an internal control. The primers used are listed as follows: PYPAF1, forward, 5'-GCCTTGAAGAGGAGTGGATAG-3' and reverse, 5'-TGGGTGTAGCGTCTGTTGAG-3'; IL-1 $\beta$, forward, 5'-TTC AAATCTCACAGCAGCAT-3' and reverse, 5'-CACGGGCAA GACATAGGTAG-3'; IL-18, forward, 5'-GCAGTAATACGG AGCATAAA-3' and reverse, 5'-ATCCTTCACAGATAGGG TCA-3'; and GAPDH, forward, 5'-ACGTTGACATCCGTAAA GAC-3' and reverse, 5'-TAGGAGCCAGGGCAGTAA-3'.

Western blot analysis. Hippocampal tissues were lysed with RIPA buffer and protein concentration was determined using a BCA protein quantification kit (both purchased from Beijing Solarbio Science and Technology Co., Ltd.). Proteins (40 $\mu \mathrm{g})$ were separated by SDS-PAGE using 8 and 14\% gels and transferred to PVDF membranes (EMD Millipore; Merck KGaA). After blocking with 5\% skimmed milk for $1 \mathrm{~h}$ at room temperature, PVDF membranes were incubated with primary antibodies at $4^{\circ} \mathrm{C}$ overnight, followed by incubation with secondary antibodies at $37^{\circ} \mathrm{C}$ for $1 \mathrm{~h}$. Subsequently, the protein bands were visualized using ECL reagent (Beijing Solarbio Science and Technology Co., Ltd.) and semi-quantified using Gel-Pro-Analyzer 4 (Media Cybernetics, Inc.). PYPAF1 antibody (1:1,000; cat. no. A5652; ABclonal Biotech Co., Ltd.), ASC antibody (1:1,000; cat. no. A1170; ABclonal Biotech Co., Ltd.), Bcl-2 antibody (1:2,000; cat. no. 12789-1-AP; Wuhan Sanying Biotechnology), Bax antibody (1:5,000; cat. no. 50599-2-1g; Wuhan Sanying Biotechnology), cleaved caspase-3 antibody (1:1,000; cat. no. 9654; Cell Signaling Technology, Inc.) and GAPDH antibody (1:10,000; cat. no. 60004-1-Ig; Wuhan Sanying Biotechnology) were the primary antibodies used. The secondary antibodies used were horseradish peroxidase (HRP)-conjugated goat anti-rabbit $\lg$ (1:3,000; cat. no. SE134; Beijing Solarbio Science and Technology Co., Ltd.) and HRP-conjugated goat anti-mouse $\operatorname{lgG}(1: 3,000$; cat. no. SE131; Beijing Solarbio Science and Technology Co., Ltd.).

Immunofluorescence assay. Hippocampal samples were embedded in paraffin and sliced into $5-\mu \mathrm{m}$ sections. The sections were dehydrated at $60^{\circ} \mathrm{C}$ in an incubator for $2 \mathrm{~h}$, deparaffinized and heated in citric acid/sodium citrate solution at $95^{\circ} \mathrm{C}$ for $10 \mathrm{~min}$. After blocking with goat serum (cat. no. SL038; Beijing Solarbio Science and Technology Co., Ltd.) for $15 \mathrm{~min}$ at room temperature, tissue sections were washed and incubated with PYPAF1 antibody (1:200; cat. no. NBP2-12446SS; Novus Biologicals, LLC) and ionized calcium binding adapter molecule-1 (Iba-1) antibody (1:200; cat.no. ab15690; Abcam) at $4^{\circ} \mathrm{C}$ overnight. Tissue sections were washed with PBS three times, incubated with FITC-labeled goat anti-mouse IgG (1:200; cat. no. A0568; Beyotime Institute of Biotechnology) and Cy3 labeled goat anti-rabbit IgG (1:200; cat. no. A0516; Beyotime Institute of Biotechnology) for $90 \mathrm{~min}$ at room temperature, and incubated with DAPI at room temperature for $5 \mathrm{~min}$ (Beyotime Institute of Biotechnology). Tissue sections were sealed with anti-fluorescence quenching reagent (Beijing Solarbio Science and Technology Co., Ltd.) and images were obtained using a fluorescence microscope (x400, magnification).

TUNEL-NeuN staining. Hippocampal sections were deparaffinized and incubated with $0.1 \%$ Triton X-100 for 8 min at room temperature (Beyotime Institute of Biotechnology). After retrieval with citric acid/sodium citrate solution at $95^{\circ} \mathrm{C}$ for $10 \mathrm{~min}$, tissues were incubated with TUNEL solution (enzyme solution:label solution, 1:9; Roche Diagnostics) for $60 \mathrm{~min}$ at $37^{\circ} \mathrm{C}$, then with NeuN antibody (1:300; cat. no. ab104224; Abcam) at $4^{\circ} \mathrm{C}$ overnight. Samples were then washed with PBS three times and incubated with Cy3 labeled goat anti-mouse IgG (1:200; cat. no. A0521; Beyotime Institute of Biotechnology) for $60 \mathrm{~min}$ at room temperature. Tissues were sealed with anti-fluorescence quenching reagent (Beijing Solarbio Science and Technology Co., Ltd.) and images were obtained using a fluorescence microscope (x400, magnification). Three sections from each sample and three fields of view in each section were randomly selected for observation, and the average values were used as the final data.

Morris water maze. The Morris water maze was performed 7 days after isoflurane exposure to assess the spatial learning and memory ability of rats. The water maze was composed of a circular tub (diameter, $180 \mathrm{~cm}$ ) and a colorless escape platform (diameter, $12 \mathrm{~cm}$ ) submerged $2 \mathrm{~cm}$ below the water surface. Rats were placed into the water from different quadrants up against the wall, and the time it took to escape was recorded. If rats failed to identify the platform within $120 \mathrm{sec}$, they were guided to the platform and allowed to rest for $15 \mathrm{sec}$. Rats were subjected to the training from different quadrants four times a day, for 5 days. Subsequently, the platform was removed on the 6th day of the trial, and the escape time, times of platform crossing and retention time at the target quadrant were recorded.

ELISA. Rat hippocampal tissues were homogenized on ice in saline to extract protein and protein concentration was quantified using a BCA protein quantification kit (Beijing Solarbio Science and Technology Co., Ltd.). The amount of IL-1 $\beta$ and IL-18 was determined using a commercial IL-1 $\beta$ ELISA kit [cat. no. 70-EK301B/3-96; MultiSciences (Lianke) Biotech Co. Ltd.] and IL-18 ELISA kit (cat. no. SEA064Ra; Wuhan USCN Business Co., Ltd.), according to the manufacturers' instructions.

Caspase-1 activity detection. Hippocampal tissue (10 mg) was lysed in $100 \mu \mathrm{l}$ lysis buffer and protein concentration was quantified using a Bradford protein assay kit (Beyotime Institute of Biotechnology). Caspase-1 activity was determined by measuring the optical density values at $405 \mathrm{~nm}$ using a commercial caspase-1 activity assay kit (cat. no. BC3810; Beijing Solarbio Science and Technology Co., Ltd.), according to the manufacturer's protocol. 
A

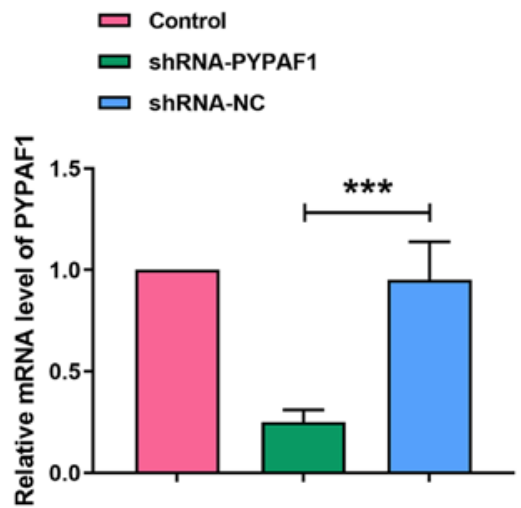

B

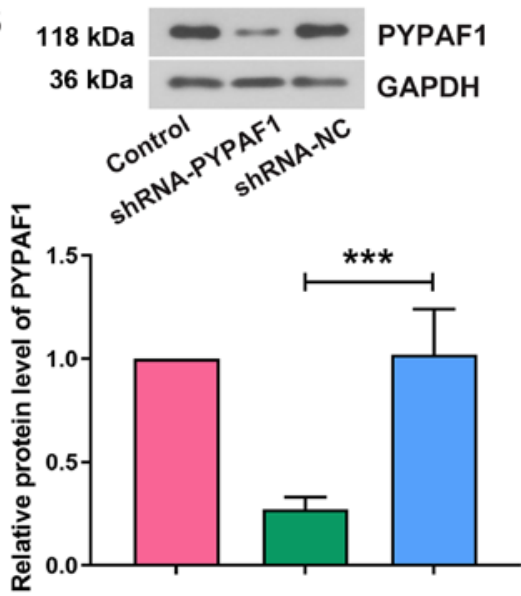

Figure 1. PYPAF1-targeting shRNA mediates the downregulation of PYPAF1 expression in the hippocampus. Sprague-Dawley rats were injected with lentivirus carrying PYPAF1-shRNA or NC-shRNA by intracerebroventricular injection. (A) mRNA and (B) protein levels of PYPAF1 in the hippocampus were measured using reverse transcription-quantitative PCR and western blot analysis, respectively. ${ }^{* * *} \mathrm{P}<0.001$. NC, negative control; PYPAF1, PYRIN-containing Apaf1-like protein 1; shRNA, short hairpin RNA.

A

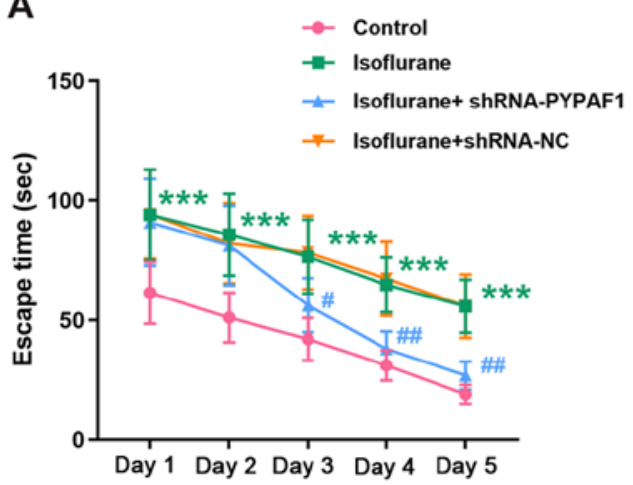

B
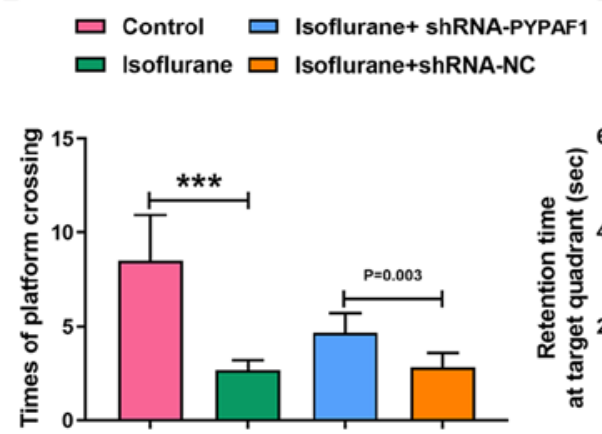

Figure 2. PYPAF1 silencing alleviates cognitive dysfunction induced by isoflurane in rats. The spatial learning ability of rats was assessed using the Morris water maze. (A) Escape time, ${ }^{* * *} \mathrm{P}<0.001$ vs. control; ${ }^{\#} \mathrm{P}<0.05,{ }^{\# \#} \mathrm{P}<0.01$ vs. isoflurane + shRNA NC. (B) Times of platform crossing and (C) retention time at target quadrant were recorded and analyzed. ${ }^{* * *} \mathrm{P}<0.001$. NC, negative control; PYPAF1, PYRIN-containing Apaf1-like protein 1; shRNA, short hairpin RNA.

Statistical analysis. Data are presented as the mean $\pm \mathrm{SD}$ and were analyzed using GraphPad Prism v7 (GraphPad Software, Inc.). The escape time in the Morris water maze was analyzed using two-way mixed ANOVA followed by post hoc Tukey test. The remaining data, including the escape time in each day, were compared using one-way ANOVA followed by post hoc Tukey test. All experiments were repeated at least three times and $\mathrm{P}<0.05$ was considered to indicate a statistically significant difference.

\section{Results}

PYPAFl expression is successfully silenced by shRNA$P Y P A F 1$. The expression of PYPAF1 in the hippocampus was detected 3 days after lentiviral infection to confirm the efficiency of RNA interference (RNAi). The mRNA expression levels of PYPAF1 were significantly lower in the shRNA-PYPAF1 group compared with in the shRNA-NC group (Fig. 1A). Western blot analysis revealed similar results (Fig. 1B), indicating that the expression of PYPAF1 was successfully knocked down in the hippocampus of aged rats.
PYPAF1 silencing alleviates the cognitive dysfunction induced by isoflurane in rats. The Morris water maze was performed to evaluate the spatial learning ability of rats 7 days after isoflurane treatment. Escape time of rats in the isoflurane group was significantly increased compared with the control group throughout the experiment $(\mathrm{P}<0.001)$. shRNA-NC did not apparently change the escape time of isoflurane-exposed rats. However, PYPAF1-targeting shRNA markedly shortened the escape time of isoflurane-exposed rats compared with the isoflurane + shRNA-NC group from day $3(\mathrm{P}<0.05$ at day 3 ; $\mathrm{P}<0.01$ at days 4 and 5; Fig. 2A). Times of platform crossing and retention time at the target quadrant were recorded on day 6 of the Morris water maze test. Rats subjected to isoflurane exposure had a lower number of platform crossings compared with rats in the control group, while PYPAF1 silencing increased the number of platform crossings (Fig. 2B). Furthermore, rats exposed to isoflurane spent less time at the target quadrant compared with rats in the control group, while silencing of PYPAF1 increased the retention time (Fig. 2C).

PYPAF1 silencing inhibits the activation of microglia. The expression levels of PYPAF1 and ASC were determined in the 
A

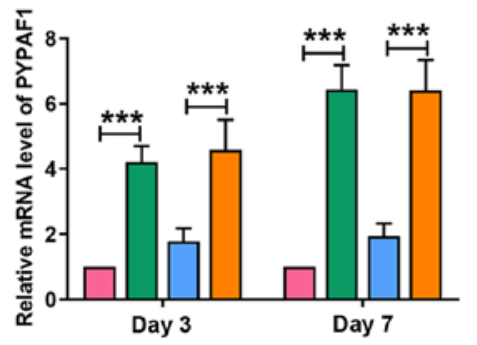

C

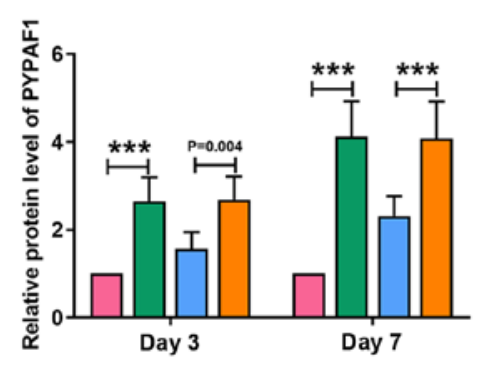

B

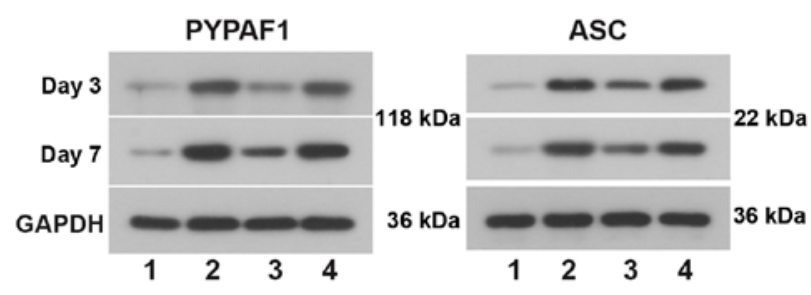

D
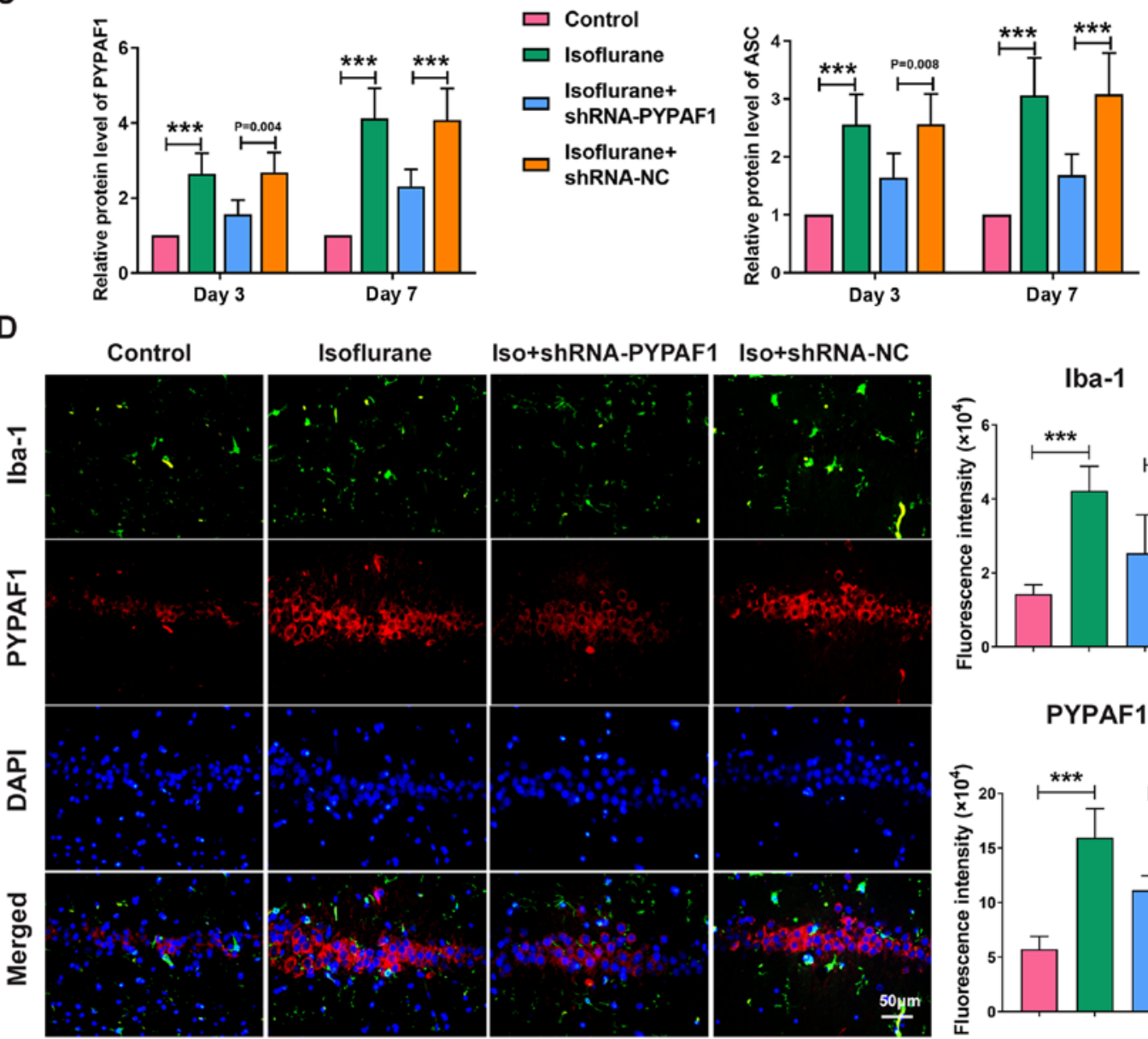

Iba-1
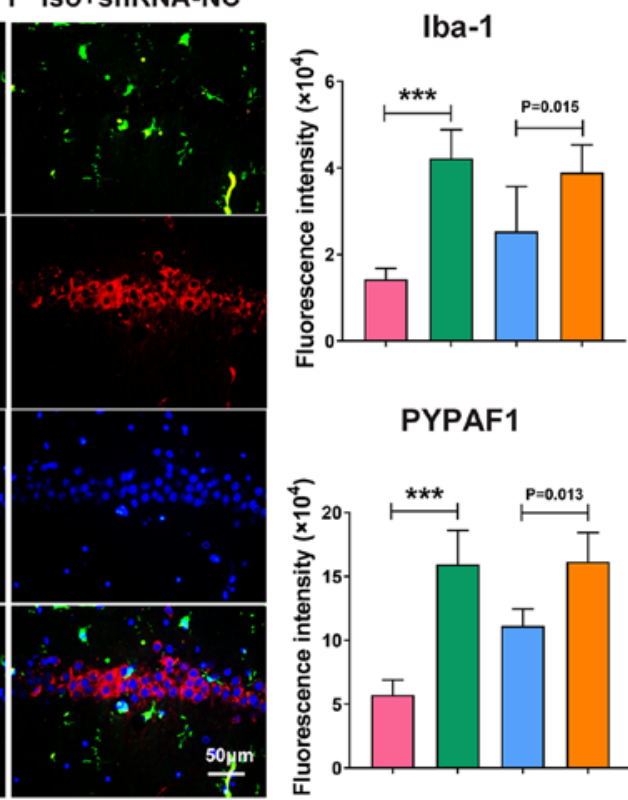

PYPAF1

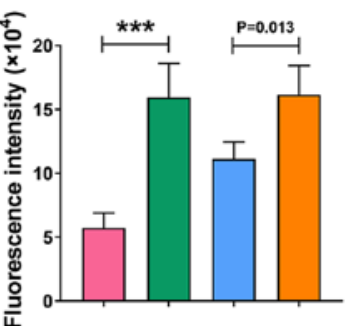

Figure 3. PYPAF1 silencing inhibits the activation of microglia. (A) mRNA levels of PYPAF1, and (B and C) protein levels of PYPAF1 and ASC in the hippocampus were measured using reverse transcription-quantitative PCR and western blot analysis on days 3 and 7 following isoflurane treatment, respectively. (B) Lane 1, control; lane 2, isoflurane; lane 3, isoflurane + shRNA-PYPAF1; lane 4, isoflurane + shRNA-NC. (D) Expression of PYPAF1 and Iba-1 in the hippocampus were determined using immunofluorescence assay. ${ }^{* * * *} \mathrm{P}<0.001$. ASC, apoptosis-associated speck-like protein containing a caspase recruitment domain; Iba-1, ionized calcium binding adapter molecule-1; Iso, isoflurane; NC, negative control; PYPAF1, PYRIN-containing Apaf1-like protein 1; shRNA, short hairpin RNA.

hippocampus on days 3 and 7 following isoflurane exposure. The mRNA expression levels of PYPAF1 were significantly higher in rats exposed to isoflurane compared with the control group, while PYPAF1 silencing using a lentivirus decreased the levels of PYPAF1 on day 3. Similarly, significant associations were observed on day 7 following isoflurane treatment (Fig. 3A). Isoflurane treatment significantly increased the protein expression levels of PYPAF1 and ASC on day 3, whereas PYPAF1 silencing partially abrogated the increase and the trend was more notable on day 7 following isoflurane exposure (Fig. 3B and C). Furthermore, the immunofluorescence assay revealed that isoflurane exposure increased the expression of Iba-1, which was significantly alleviated by knockdown of PYPAF1 (Fig. 3D).
PYPAF1 silencing inhibits neuroinflammation. RT-qPCR and ELISA were performed to determine the levels of IL-1 $\beta$ and IL-18. The mRNA expression and concentration of IL-1 $\beta$ and IL-18 in the hippocampus were increased following isoflurane exposure, whereas PYPAF1 silencing decreased their levels (Fig. 4A and B). Furthermore, isoflurane treatment enhanced the activity of caspase-1, which was partially abolished by silencing PYPAF1 (Fig. 4C). These results indicated that silencing of PYPAF1 inhibited neuroinflammation in rats.

PYPAF1 silencing alleviates neuronal apoptosis. TUNEL assay was performed to assess neuronal apoptosis in the hippocampus. Apoptotic neurons were markedly increased 
A B

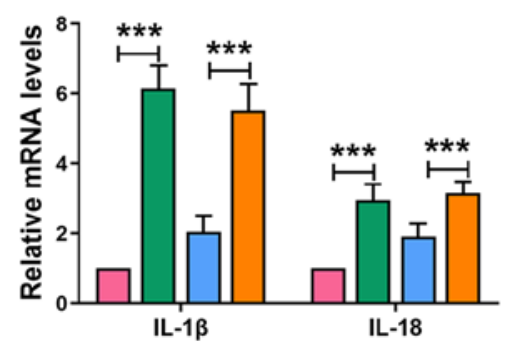

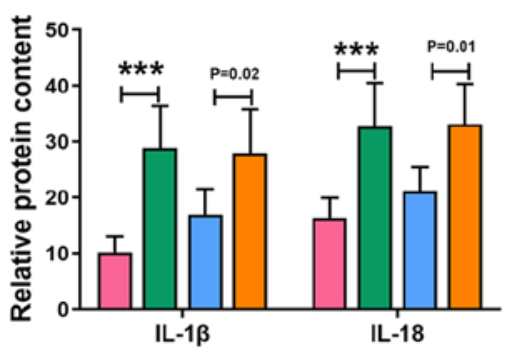

C $\square$ Control $\square$ Isoflurane+ shRNA-PYPAF1

$\square$ Isoflurane $\square$ Isoflurane+shRNA-NC

Figure 4. PYPAF1 knockdown inhibits the activity of caspase-1, and expression of IL-1 $\beta$ and IL-18. (A) mRNA levels and (B) concentration of IL-1 $\beta$ and IL-18 in the hippocampus was determined using reverse transcription-quantitative PCR and ELISA, respectively, on day 3 following isoflurane treatment. (C) Activity of caspase-1 in the hippocampus was assessed using a commercial caspase-1 activity assay kit on day 3 following isoflurane treatment. ${ }^{* * *} \mathrm{P}<0.001$. IL, interleukin; NC, negative control; PYPAF1, PYRIN-containing Apaf1-like protein 1; shRNA, short hairpin RNA.
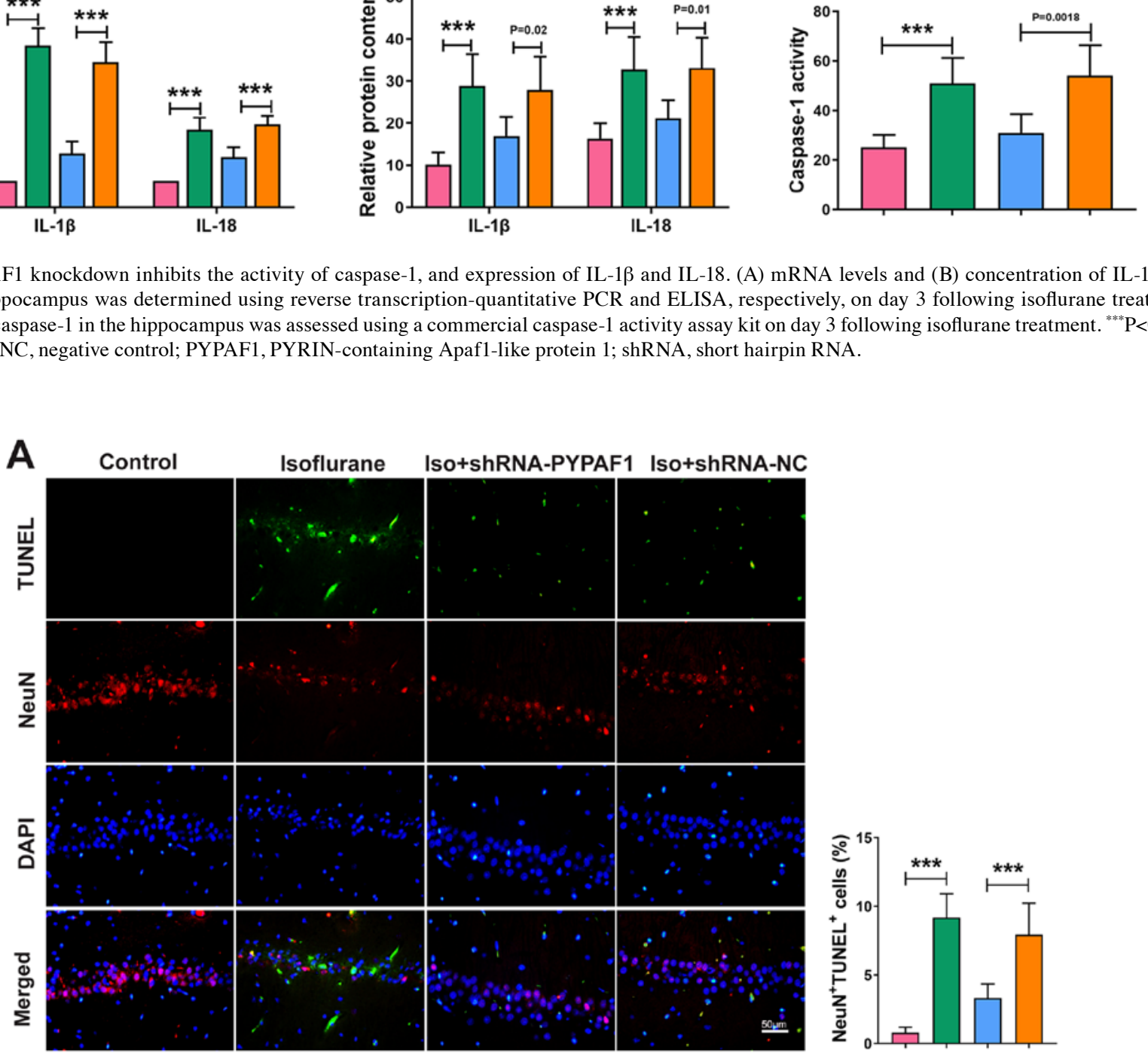

B

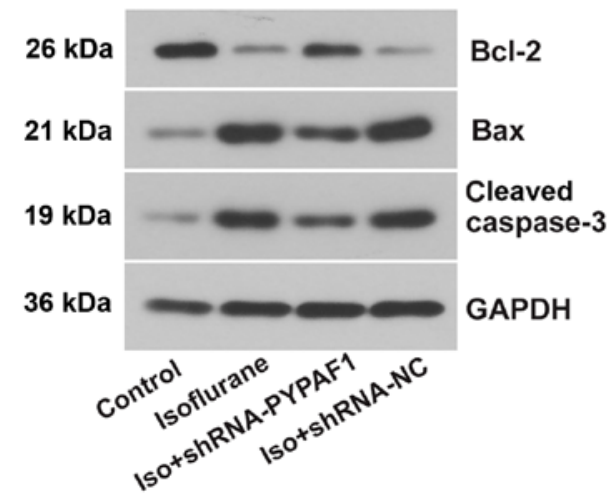

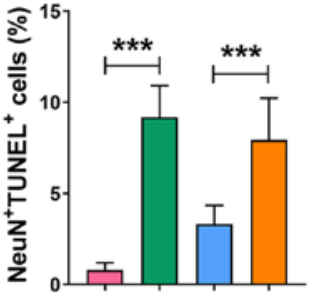

$\square$ Control $\square$ Isoflurane+ shRNA-PYPAF1

$\square$ Isoflurane $\square$ Isoflurane+shRNA-NC

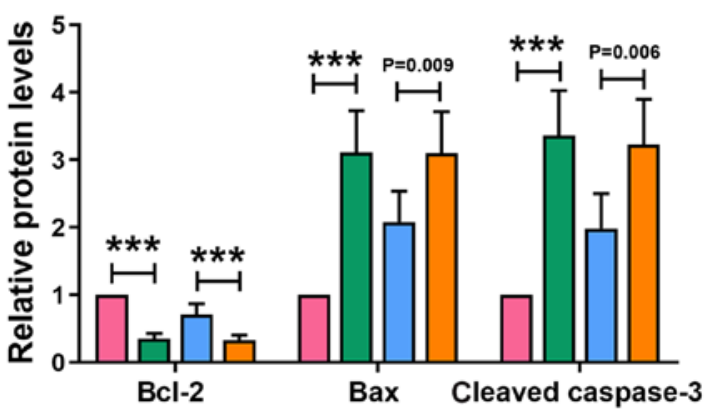

Figure 5. PYPAF1 silencing alleviates neuronal apoptosis. (A) Neuronal apoptosis in the hippocampus was determined using TUNEL-NeuN double staining on day 3 following isoflurane treatment. (B) Protein expression levels of Bcl-2, Bax and cleaved caspase-3 in the hippocampus were determined using western blot analysis. ${ }^{* * *} \mathrm{P}<0.001$. Iso, isoflurane; NC, negative control; PYPAF1, PYRIN-containing Apaf1-like protein 1; shRNA, short hairpin RNA.

following isoflurane exposure, which was partially reversed following PYPAF1 knockdown. Isoflurane treatment decreased the expression of NeuN, whereas silencing PYPAF1 alleviated the effects of isoflurane (Fig. 5A). Furthermore, the protein expression levels of Bax and cleaved caspase-3 were increased by isoflurane, and the elevation was partially abolished by silencing of PYPAF1. The protein levels of Bcl-2 revealed the opposite results (Fig. 5B). 


\section{Discussion}

In the present study, isoflurane upregulated the expression levels of PYPAF1 in the hippocampus of aged rats. RNAi-mediated PYPAF1 knockdown significantly improved learning and memory function of isoflurane-exposed aged rats. In addition, PYPAF1 knockdown inhibited activation of microglia, production of proinflammatory cytokines and neuronal apoptosis in the hippocampus of isoflurane-exposed aged rats.

Isoflurane is a widely used inhalational anesthetic agent owing to its low cost and relatively few side effects (26). Inhaled anesthesia could induce cerebral cognitive alterations in elderly patients $(10,27)$. Furthermore, a clinical trial involving elderly patients that were subjected to laparoscopic cholecystectomy revealed that patients who received isoflurane anesthesia displayed a significantly higher incidence of POCD compared with those who received sevoflurane and propofol $(10,28)$. In the present study, the spatial learning ability of rats exposed to isoflurane was markedly reduced, which was accompanied by increased expression of PYPAF1 and ASC, whereas shRNA-mediated PYPAF1 knockdown markedly alleviated the cognitive dysfunction of aged rats and decreased the expression of ASC. The PYPAF1 inflammasome is abundantly expressed in the CNS and activation of the PYPAF1 inflammasome is involved in the pathogenesis of neurological diseases, including AD and depression $(29,30)$. PYPAF1 is the cytosolic sensor molecule of the PYPAF1 inflammasome and abundant expression of PYPAF1 is crucial for inflammasome formation (21). ASC activates the PYPAF1 inflammasome by recruiting the cysteine protease pro-caspase-1 via its caspase recruitment domain (31). In the present study, shRNA-mediated PYPAF1 knockdown hindered the assembling of the PYPAF1 inflammasome and suppressed the expression of ASC. Furthermore, isoflurane exposure led to the activation of caspase-1, which was also inhibited following knockdown of PYPAF1. PYPAF1 silencing also abolished isoflurane-induced elevated IL-1 $\beta$ and IL-18 at both the mRNA and protein levels. These findings are consistent with previous reports, which indicated that activation of the PYPAF1 inflammasome further activated inflammatory protease caspase-1, and promoted the secretion of pro-inflammatory cytokines IL-1 $\beta$ and IL-18 $(21,32)$. Increased levels of IL-1 $\beta$ and IL-18 may trigger a series of signaling pathways and inflammatory responses, which could lead to neuronal injury or apoptosis, whereas knockdown of IL-1 $\beta$ in mice significantly alleviated neuroinflammatory and memory dysfunction following surgery and anesthesia $(33,34)$. The results from the present study indicated that the PYPAF1/ASC pathway may contribute to isoflurane-induced POCD in aged rats.

Microglia, a primary effector cell that responds to pathological challenges in the CNS, serves a crucial role in neuroprotection and in the maintenance of hemostasis (35). Mild activation of microglia exerts a protective effect on the CNS, whereas its over-activation results in neuronal dysfunction $(36,37)$. Furthermore, microglia express numerous PRRs that are essential for assembling the inflammasome (38). In the present study, isoflurane exposure led to activation of microglia in the hippocampus of aged rats, and PYPAF1 knockdown inhibited the activation of microglia, as evidenced by the decrease in the expression of Iba-1. It is well known that microglial activation occurs in the early stage of neuroinflammation, which leads to the production of proinflammatory cytokines and induces cognitive dysfunction (39). In addition, aging causes microglia to be more susceptible to activation (40). The findings from the present study indicated that PYPAF1 may have a critical role in microglial activation following isoflurane exposure. However, the present study has not revealed whether this inhibitory effect on microglial activation is direct or indirect; therefore, further investigation is required in future studies.

Neuronal function is the foundation of learning and memory (41). In the present study, isoflurane exposure induced neuronal apoptosis and decreased NeuN expression in the rat hippocampus, whereas PYPAF1 silencing alleviated neuronal apoptosis and elevated the levels of NeuN. Isoflurane exposure elevated the expression of pro-apoptotic factor, Bax, and cleaved caspase-3, and decreased the expression of the anti-apoptotic factor, Bcl-2. Furthermore, silencing of PYPAF1 abrogated isoflurane-induced neuronal apoptosis. These results suggested that PYPAF1 knockdown may inhibit neuronal apoptosis in the hippocampus of aged rats exposed to isoflurane. However, whether PYPAF1-targeting shRNA directly affects apoptotic pathways, or inhibits apoptosis acts via its effect on inflammasome inhibition, requires further investigation. The role that the PYPAF1 inflammasome plays in the development of POCD has not been well reported. Wang et al (42) revealed that isoflurane exposure induced cognitive impairment and neuroinflammation, which are more likely to occur in aged mice, and a caspase- 1 inhibitor mitigated cognitive dysfunction. In addition, knockdown of PYPAF1 in BV-2 cells inhibited PYPAF1 inflammasome activation and IL-1 $\beta$ production (42). To the best of our knowledge, the present study was the first to reveal that PYPAF1 knockdown protects aged rats from isoflurane-induced cognitive impairment, which may be associated with the effect on inhibition of microglial activation and neuronal apoptosis in the hippocampus.

There are limitations to the present study. Firstly, analysis of the hippocampus and proinflammatory factors were examined 3 and 7 days following exposure to isoflurane. The changes in PYPAF1 expression at earlier and later time points will be observed in future studies. Secondly, the exact role of PYPAF1 in different types of brain cells, such as microglia and neurons, under the exposure of isoflurane was not determined, as these could not be isolated and cultured from aged animals.

In conclusion, the findings from the present study demonstrated that PYPAF1 knockdown alleviated isoflurane-induced cognitive impairment in aged rats. Neuroinflammation and neuronal apoptosis inhibition may contribute to this effect. Therefore, the PYPAF1 inflammasome may serve as a potential therapeutic target for the treatment of POCD.

\section{Acknowledgements}

Not applicable.

\section{Funding}

No funding was received. 


\section{Availability of data and materials}

The datasets used and/or analyzed during the current study are available from the corresponding author on reasonable request.

\section{Authors' contributions}

YZ designed the study. XZ, XF, FL and JQ contributed to the acquisition, analysis and interpretation of data. $\mathrm{XZ}$ drafted the article. YZ critically revised it for important intellectual content. All authors read and approved the final manuscript.

\section{Ethics approval and consent to participate}

All animal experiments were performed at the animal center at the First Hospital of Jilin University following the guidelines for the care and use of laboratory animals, and the study was approved by the First Hospital of Jilin University.

\section{Patient consent for publication}

Not applicable.

\section{Competing interests}

The authors declare that they have no competing interests.

\section{References}

1. Hartholt KA, van der Cammen TJ and Klimek M: Postoperative cognitive dysfunction in geriatric patients. Z Gerontol Geriatr 45: 411-416, 2012.

2. Rundshagen I: Postoperative cognitive dysfunction. Dtsch Arztebl Int 111: 119-125, 2014.

3. Rudolph JL, Marcantonio ER, Culley DJ, Silverstein JH, Rasmussen LS, Crosby GJ and Inouye SK: Delirium is associated with early postoperative cognitive dysfunction. Anaesthesia 63: 941-947, 2008.

4. Nadelson MR, Sanders RD and Avidan MS: Perioperative cognitive trajectory in adults. Br J Anaesth 112: 440-451, 2014

5. Polunina AG, Golukhova EZ, Guekht AB and Lefterova NP and Bokeria LA: Cognitive dysfunction after on-pump operations: neuropsychological characteristics and optimal core battery of tests. Stroke Res Treat 2014: 302824, 2014.

6. Saczynski JS, Inouye SK, Kosar C, Tommet D, Marcantonio ER, Fong T, Hshieh T, Vasunilashorn S, Metzger ED, Schmitt E, et al: Cognitive and brain reserve and the risk of postoperative delirium in older patients. Lancet Psychiatry 1: 437-443, 2014.

7. Krenk L, Rasmussen LS and Kehlet H: New insights into the pathophysiology of postoperative cognitive dysfunction. Acta Anaesthesiol Scand 54: 951-956, 2010.

8. Ministry of Civil Affairs of the People's Republic of China: The social service development statistical bulletin 2018.http://images3 mca.gov.cn/www2017/file/201908/1565920301578.pdf.Accessed Feb 13, 2020

9. Kincannon CL, He W and West LA: Demography of aging in China and the United States and the economic well-being of their older populations. J Cross Cult Gerontol 20: 243-255, 2005.

10. Mandal PK, Schifilliti D, Mafrica F and Fodale V: Inhaled anesthesia and cognitive performance. Drugs Today (Barc) 45: 47-54, 2009.

11. Amor S and Woodroofe MN: Innate and adaptive immune responses in neurodegeneration and repair. Immunology 141: 287-291, 2014.

12. Li N, Zhang X, Dong H, Hu Y and Qian Y: Bidirectional relationship of mast cells-neurovascular unit communication in neuroinflammation and its involvement in POCD. Behav Brain Res $322: 60-69,2017$

13. Wu X, Lu Y, Dong Y, Zhang G, Zhang Y, Xu Z, Culley DJ, Crosby G, Marcantonio ER, Tanzi RE, et al: The inhalation anesthetic isoflurane increases levels of proinflammatory TNF- $\alpha$, IL-6, and IL-13. Neurobiol Aging 33: 1364-1378, 2012.
14. Sierra A, Gottfried-Blackmore AC, McEwen BS and Bulloch K: Microglia derived from aging mice exhibit an altered inflammatory profile. Glia 55: 412-424, 2007.

15. Hansen DV, Hanson JE and Sheng M: Microglia in Alzheimer's disease. J Cell Biol 217: 459-472, 2018.

16. Qiu LL, Ji MH, Zhang H, Yang JJ, Sun XR, Tang H, Wang J, Liu WX and Yang JJ: NADPH oxidase 2-derived reactive oxygen species in the hippocampus might contribute to microglial activation in postoperative cognitive dysfunction in aged mice. Brain Behav Immun 51: 109-118, 2016.

17. Wang HL, Ma RH, Fang H, Xue ZG and Liao QW: Impaired spatial learning memory after isoflurane anesthesia or appendectomy in aged mice is associated with microglia activation. J Cell Death 8: 9-19, 2015.

18. Lamkanfi M and Dixit VM: Mechanisms and functions of inflammasomes. Cell 157: 1013-1022, 2014.

19. Song L, Pei L, Yao S, Wu Y and Shang Y: NLRP3 inflammasome in neurological diseases, from functions to therapies. Front Cell Neurosci 11: 63, 2017.

20. Manji GA, Wang L, Geddes BJ, Brown M, Merriam S, Al-Garawi A, Mak S, Lora JM, Briskin M, Jurman M, et al: PYPAF1, a PYRIN-containing Apaf1-like protein that assembles with ASC and regulates activation of NF-kappa B. J Biol Chem 277: 11570-11575, 2002.

21. Próchnicki T, Mangan MS and Latz E: Recent insights into the molecular mechanisms of the NLRP3 inflammasome activation. F1000Res 5: F1000, 2016.

22. Zhang N, Zhang X, Liu X, Wang H, Xue J, Yu J, Kang N and Wang X: Chrysophanol inhibits NALP3 inflammasome activation and ameliorates cerebral ischemia/reperfusion in mice. Mediators Inflamm 2014: 370530, 2014.

23. Zhang Z, Li X, Li F and An L: Berberine alleviates postoperative cognitive dysfunction by suppressing neuroinflammation in aged mice. Int Immunopharmacol 38: 426-433, 2016.

24. National Academy of Sciences: The National Academies Collection: Reports funded by National Institutes of Health. National Academies Press, Washington, DC, 1975.

25. Livak KJ and Schmittgen TD: Analysis of relative gene expression data using real-time quantitative PCR and the 2(-Delta Delta C(T)) method. Methods 25: 402-408, 2001.

26. Powell LM and Molyneux M: Should patients be advised not to drive for 4 days after isoflurane anaesthesia? Anaesthesia 72: 682-685, 2017.

27. Mahajan VA, Ni Chonghaile M, Bokhari SA, Harte BH, Flynn NM and Laffey JG: Recovery of older patients undergoing ambulatory anaesthesia with isoflurane or sevoflurane. Eur J Anaesthesiol 24: 505-510, 2007.

28. Geng YJ, Wu QH and Zhang RQ: Effect of propofol, sevoflurane, and isoflurane on postoperative cognitive dysfunction following laparoscopic cholecystectomy in elderly patients: A randomized controlled trial. J Clin Anesth 38: 165-171, 2017.

29. Kaufmann FN, Costa AP, Ghisleni G, Diaz AP, Rodrigues AL, Peluffo $\mathrm{H}$ and Kaster MP: NLRP3 inflammasome-driven pathways in depression: Clinical and preclinical findings. Brain Behav Immun 64: 367-383, 2017.

30. White CS, Lawrence CB, Brough D and Rivers-Auty J: Inflammasomes as therapeutic targets for Alzheimer's disease. Brain Pathol 27: 223-234, 2017.

31. Proell M, Gerlic M, Mace PD, Reed JC and Riedl SJ: The CARD plays a critical role in ASC foci formation and inflammasome signalling. Biochem J 449: 613-621, 2013.

32. Martinon F, Burns K and Tschopp J: The inflammasome: A molecular platform triggering activation of inflammatory caspases and processing of prolL-beta. Mol Cell 10: 417-426, 2002.

33. Yatsiv I, Morganti-Kossmann MC, Perez D, Dinarello CA, Novick D, Rubinstein M, Otto VI, Rancan M, Kossmann T, Redaelli CA, et al: Elevated intracranial IL-18 in humans and mice after traumatic brain injury and evidence of neuroprotective effects of IL-18-binding protein after experimental closed head injury. J Cereb Blood Flow Metab 22: 971-978, 2002.

34. Cibelli M,Fidalgo AR, Terrando N,MaD, Monaco C,Feldmann M, Takata M, Lever IJ, Nanchahal J, Fanselow MS, et al: Role of interleukin-1beta in postoperative cognitive dysfunction. Ann Neurol 68: 360-368, 2010.

35. Nayak D, Roth TL and McGavern DB: Microglia development and function. Annu Rev Immunol 32: 367-402, 2014.

36. Lalancette-Hébert M, Gowing G, Simard A, Weng YC and Kriz J: Selective ablation of proliferating microglial cells exacerbates ischemic injury in the brain. J Neurosci 27: 2596-2605, 2007. 
37. Moss DW and Bates TE: Activation of murine microglial cell lines by lipopolysaccharide and interferon-gamma causes NO-mediated decreases in mitochondrial and cellular function. Eur J Neurosci 13 529-538, 2001.

38. Ghosh S, Castillo E, Frias ES and Swanson RA: Bioenergetic regulation of microglia. Glia 66: 1200-1212, 2018.

39. Fernandes A, Miller-Fleming L and Pais TF: Microglia and inflammation: Conspiracy, controversy or control? Cell Mol Life Sci 71: 3969-3985, 2014.

40. Fougère B, Boulanger E, Nourhashémi F, Guyonnet $S$ and Cesari M: Chronic Inflammation: Accelerator of Biological Aging. J Gerontol A Biol Sci Med Sci 72: 1218-1225, 2017.
41. Kennedy MB: Synaptic signaling in learning and memory. Cold Spring Harb Perspect Biol 8: a016824, 2013.

42. Wang Z, Meng S, Cao L, Chen Y,Zuo Z and Peng S: Critical role of NLRP3-caspase-1 pathway in age-dependent isoflurane-induced microglial inflammatory response and cognitive impairment. J Neuroinflammation 15: 109, 2018.

(i) (9) This work is licensed under a Creative Commons cc) Attribution-NonCommercial-NoDerivatives 4.0 International (CC BY-NC-ND 4.0) License. 